\title{
Hepatitis C: improving the quality of screening in a community hospital by implementing an electronic medical record intervention
}

\author{
Vahe Shahnazarian, Eric Karu, Parag Mehta \\ New York Methodist Hospital, USA
}

\begin{abstract}
Both the Centers for Disease Control and Prevention (CDC) and the U.S. Preventive Services Task Force (USPSTF) have recommended that adults born between the years of 1945-1965 should receive one-time testing for Hepatitis C Virus (HCV). In fact, Governor Andrew Cuomo of the State of New York had signed a bill on October 23, 2013 which mandated NY hospitals and healthcare providers to offer HCV testing to all "Baby Boomers." For our project, we wanted to increase our community hospital's compliance with this law and improve the quality of patient care in doing so.
\end{abstract}

An electronic medical record intervention was implemented in conjunction with our information technology services department. This intervention would flag eligible patients and would run them through a predetermined algorithm to see if they needed HCV testing. Multiple plan, do, study, act (PDSA) cycles were run during the length of the study and many changes were made in order to achieve maximum effect.

We ended up increasing our HCV testing rate from $47.2 \%$ (pre-intervention) to $87.9 \%$ (final month of the study), which was statistically significant with a p-value of $<0.0000001$. We also ended up with a framework that is both generalizable to other projects and is also selfsustaining, so that it can continue to run itself once all the project members have finished working there as house staff.

\section{Problem}

Hepatitis $C$ testing was not appropriately being offered at New York Methodist Hospital in Brooklyn, New York, USA. We realized this after speaking to resident physicians and attendings at the hospital. A majority of them either said they did not fully know the recommended guidelines or that they were altogether forgetting to offer or order the testing. We actually ran the numbers and it was noted that only $47 \%$ of eligible patients were having the testing offered to them. This was far too low of a number, especially given the fact that the Governor of New York State had recently passed a law which mandated offering testing to Baby Boomers. We wanted to address this problem in the most effective and efficient way possible.

\section{Background}

Hepatitis $\mathrm{C}$ virus (HCV) is the most common chronic blood-borne pathogen in the United States and a leading cause of complications from chronic liver disease.[1] In the United States, approximately $1.6 \%$ of non-institutionalized adults are positive for the Hepatitis $C$ antibody.[1] Also, people born between the years of 1945-1965 ("Baby Boomers") have a hepatitis C antibody prevalence of $3.25 \%$, which is five times higher than that of adults born in other years, and accounts for almost three quarters of all the hepatitis $C$ infections in the United States. Men have double the prevalence of women, and the highest prevalence is among non-Hispanic black males, at $8.12 \%$.[2] The most important risk factor for $\mathrm{HCV}$ is current or past intravenous drug abuse (IVDA).[1]
Adults born between 1945 and 1965 are more likely to be diagnosed with HCV infection, either because they received a blood transfusion before the introduction of screening in 1992 or because they have a history of other risk factors for exposure decades earlier.[2] Other risk factors include: incarceration, intranasal drug abuse, long-term hemodialysis, getting an unregulated tattoo, other percutaneous exposures, and being born to a mother that is infected with hepatitis C.[1] Also, it is important to note that while an $\mathrm{HCV}$ infection can be characterized from mild to severe, many people are asymptomatic and do not know they are infected with HCV.[1,2]

As a result of this information, both the Centers for Disease Control and Prevention (CDC) and the U.S. Preventive Services Task Force (USPSTF) have recommended that adults born between the years of 1945-1965 should receive one-time testing for HCV (CDC B recommendation and USPSTF strong recommendation with moderate quality of evidence).[1,2] In fact, Governor Andrew Cuomo of the State of New York had signed a bill on October 23, 2013 which mandated NY hospitals and healthcare providers to offer HCV testing to all Baby Boomers. The law excludes the emergency department. This law had taken effect on January 1 , 2014.[3]

The importance of this law lies in the fact that people who have HCV and are identified early on can benefit from early treatment and avoid liver complications.[1] The later the HCV infection is found, the lower the chance that the treatment will have a sustained response.[2] Also, HCV-associated disease is the number one cause of liver transplantation and hepatocellular carcinoma (HCC) in the United States. The incidence of HCC and transplant are 
increasing in people who have HCV, and these numbers are projected to substantially increase in the coming decade.[2] Given the advancement of current HCV therapies, the interferon-free regimens will likely dominate, making treatment easier to follow, so having early, successful treatment of HCV infection has "undeniable long-term benefits with respect to reducing morbidity and mortality" in this population.[4,5]

This study aims to increase New York Methodist Hospital's compliance with the offering of state mandated hepatitis C Testing to people born between the years of 1945 and 1965 . This will be accomplished by studying patient charts and orders from before the law was passed, after the law was passed (with no intervention implemented) and then after an intervention is implemented. We hypothesize that prior to the passage of the law, included patients had a low rate of hepatitis $C$ testing. After the passage of the law, but prior to intervention, that rate will still be low. We believe that the rate of offering hepatitis $C$ testing will greatly increase with the implementation of the intervention.

\section{Baseline measurement}

Before the project was initiated, we came up with an algorithm in the electronic medical records (EMR) to measure how many admitted and clinic patients were eligible for Baby Boomer testing (born between 1945-1965). Then the program was able to tell us actually how many of those patients had an hepatitis $\mathrm{C}$ virus (HCV) test result in the computer (either antibody or PCR). In our control month of December 2013 (pre-law, pre-intervention), only $47.2 \%$ of qualified patients had been tested for HCV. In our month of January 2014 (post-law, pre-intervention), only $46.8 \%$ of patients were tested for HCV.

\section{Design}

The plan, do, study, act (PDSA) model provides methods to improve the quality of healthcare systems.[6] The cycle is as follows: in the "plan" step, objectives are stated and a detailed plan is formulated. In the "do" phase, all changes from the "plan" phase are implemented, after which detailed data is gathered. In the "study" phase, all of the gathered data is analyzed. Finally, in the "act" phase, one considers adopting, adapting or abandoning the implemented changed (depending on their levels of success). All the steps of the cycle are then integrated into the next cycle; the number of cycles depends on how quickly, if at all, the measured change is achieved.[7]

Patients included in the study were all those born between 1945 and 1965 that were either admitted to the hospital or seen in the outpatient clinic setting (the law does not require the emergency department (ED) to offer testing). Once a patient is flagged by the EMR system as being eligible, it will check to see if the patient has either ever been offered HCV testing or if they have ever received it in the past. If they have, regardless of the result, the patient is now excluded. If they have never been offered or received testing, then the EMR goes on to the next step: an action box pops-up, asking the healthcare professional to offer testing to the patient, and what the patient's response is (accepted, rejected, unable to make a decision secondary to clinical condition, or already received elsewhere). Once a selection has been made, it is documented in the EMR. If the patient refuses or has had the testing elsewhere, they are excluded from the EMR algorithm forever. If they accept or are unable to make a decision, the test is then automatically ordered by the EMR (HCV antibody testing, which has a sensitivity of $97 \%$, specificity of $100 \%$ and costs only $\$ 3.75$ per test).[5] This was the basic initial algorithm.

In order to accomplish this, we developed a list of simple initiatives, gathered an interdisciplinary team which included members of the information technology services team (IT), department of medicine (DoM), laboratory medicine team, and hospital administration, identified early adopters, and came up with a plan for rapid pilots, rapid feedback and how to advertise gains.[7] Together, it was decided that an EMR intervention would be the most efficient and effective option in addressing our compliance issue (given the fact that a recent study showed $42 \%$ of primary care doctors are unfamiliar with the CDC guidelines for testing).[5] It took approximately three weeks of meetings, but the initial product was implemented on April 15, 2014. After this, the intervention went through multiple PDSA cycles.

\section{Strategy}

It was initially noted by our early adopters (who were giving us rapid feedback), that the pop-up was being seen in every single outpatient setting that was connected to our electronic medical records (EMR) system. This was not our intent, so we set new guidelines for the pop-up to only appear in primary care clinics in the outpatient setting (as opposed to being seen in the chemotherapy infusion center, for example).

Next, it was noted that the clinic patients were not automatically having the test ordered if they qualified and agreed. That was a simple fix by IT as well. We also realized that the people who had already received the test were not being auto-charted by the EMR. After some investigation, it was noted that the HCV PCR test and the HCV viral load test had not been added to the algorithm; it was subsequently added.

Throughout the first few weeks, during our rapid feedback, it was noted that the DoM house staff were not all clicking into the pop-up in order to offer testing to the patients. They were saying that we had so many pop-ups in the EMR that they were accidentally ignoring the pop-up so that they could quickly get into the patient's chart to do their work. The interdisciplinary team met again, and it was decided that we would change the pop-up to fire as soon as an admission order was placed by the ED; this way, the admitting resident would be responsible for asking the patient.

Again, more feedback showed that the testing was still not being done due to too many pop-ups. At that point, the New York State Department of Health was contacted in order to make sure that no consent was needed for Hepatitis $\mathrm{C}$ testing. This was confirmed, and so, in conjunction with hospital administration, the decision was made to automatically order the test for all qualified patients 
(especially in light of the fact that the oral treatment of HCV has been shown to be cost effective, especially when instituted prior to late onset morbidity associated with the disease).[5] Now, instead of having a pop-up notify you to ask for testing, a pop-up would only occur if the patient tested positive for Hepatitis C. Also, the patient's positive hepatitis $\mathrm{C}$ test would auto-populate into their discharge summary so that they could discuss it with their primary care physician and gastroenterologist; it would also print up a standard handout with information on HCV for the patient upon discharge. This final adjustment proved to be the best compromise for our rapid feedback providers.

\section{Results}

Starting from our intervention month of May 2014, we saw a statistically significant increase in our testing results. The study ran through February 2015, and we had a statistically significant increase in testing in all months except for October 2014. These results are summarized in table 1 and chart 1 .

We also wanted to study the number of newly diagnosed patients in each month. As compared to our control month of December 2013, almost every month after the intervention was implemented showed a statistically significant increase in the number of patients with newly diagnosed HCV per month. These results are summarized in table 2.

See supplementary file: ds5096.docx - "Table 1, Table 2 and Chart $1 "$

\section{Lessons and limitations}

While the intervention initially met with some difficulty, after the multiple PDSA cycles described above, it was finally fully adopted by all users in our hospital system. In fact, the feedback that we began to receive from most users had become overwhelmingly positive since we had removed the responsibility from the care provider and placed it directly on the EMR. We were able to end up using the EMR to achieve maximum effort on our outcome measure.[7]

One important point was to make note of the effect of EMR alert fatigue. This proved to play a huge role on how we altered the entire intervention. Alert fatigue has been defined as "declining clinician responsiveness to a particular type of alert as the clinician is repeatedly exposed to that alert over a period of time, gradually becoming 'fatigued' or desensitized to it."[8] We needed to find a balance between all of our EMR alerts (sirs/sepsis alert, Foley catheter renewal, diet orders, etc.) and how to ensure that the important ones would not be missed.[8] We cut down on the number of times our pop-up would occur, and this made both the house staff and attending physicians much happier with the intervention.

In completing our multiple PDSA cycles, we were able to not only address the problems with the intervention, but to also maximize its effect. In doing so, we were able to progress towards achieving our goal of $100 \%$ of qualified patients having their HCV testing done.

Also, the great thing about an EMR project is that thanks to all the work we did with the IT department, this project is completely sustainable even when I leave the residency program. It has the capability of running itself. Also, since we have created the initial framework in the EMR, this can also be applied to many other projects. It is completely generalizable to other program as well.

\section{Conclusion}

With this intervention, we were able to maximize our outcome measure of HCV testing for our qualified patients. This was successfully done by the implementation of an EMR intervention, after multiple PDSA cycles, thanks to our interdisciplinary team and early adopters.[9] They made it possible for us to have rapid feedback and were also integral in advertising our gains (mostly by word of mouth).

This study highlights the important role of healthcare leaders in driving a culture that improves quality and patient safety.[7] We were able to increase the number of patients identified as being infected with $\mathrm{HCV}$, with the hope that they will receive treatment prior to developing the late complications of this disease. This will benefit not only the patient (given all the new treatments that are available), but it will also decrease their burden on the healthcare system (since they will be treated, and likely cured).

Lastly, the Accreditation Council for Graduate Medical Education (ACGME) has acknowledged the vital importance of the clinical learning environment as part of resident education. In doing so, they have generated the clinical learning environment review (CLER) in order to further pursue these interests and to underline their importance as an integral part of graduate medical education. This quality improvement intervention helps to show that house staff can make a significant difference in the patient care rendered at their own hospitals and that they can continue to become "lifelong learners and improvers who will advance clinical improvements in patient care and system performance."[10]

\section{References}

1. Moyer VA. Screening for Hepatitis C Virus Infection in Adults: U.S. Preventive Services Task Force Recommendation Statement. Ann Intern Med 2013;159:349-57.

2. Centers for Disease Control and Prevention. Recommendations for the Identification of Chronic Hepatitis C Virus Infection Among Persons Born During 1945-1965. MMWR 2012;61(No. RR-4):1-32.

3. Governor Cuomo Signs Bill to Require Hospitals to Offer Hepatitis C Testing. Governor Andrew M. Cuomo. New York State Governor's Office. 8 Feb 2014.

4. Liang TJ, Ghany MG. Current and Future Therapies for Hepatitis C Virus Infection. New Engl J Medi 2013;368:1907-7.

5. Joshi SN. Hepatitis C Screening. Ochsner J 2014;14:664-8. 


\section{BMJ Quality Improvement Reports}

6. Taylor MJ, McNicholas C, Nicolay C et al. Systematic review of the application of the plan-do-study-act method to improve quality in healthcare. BMJ Qual Saf 2013 10.1136/bmjqs-2013-001862

7. Schriefer J, Leonard MS. Patient Safety and Quality Improvement: An Overview of QI. Pediatr Rev 2012;33:353-60.

8. Carspecken CW, Sharek PJ, Longhurst C, Pageler NM. A Clinical Case of Electronic Health Record Drug Alert Fatigue: Consequences for Patient Outcome. Pediatrics 2013;131:e1970-3.

9. Love TE, Cebul RD, Einstadter D, Jain AK, Miller H, Harris $\mathrm{CM}$, et al. Electronic Medial Record-Assisted Design of a Cluster-Randomized Trial to Improve Diabetes Care and Outcomes. J Gen Intern Med 2007;23(4):383-91.

10. Jones AC, Shipman SA, Ogrinc G. Key characteristics of successful quality improvement curricula in physician education: a realist review. BMJ Qual Saf 2015;24:77-88.

\section{Declaration of interests}

Nothing to declare.

\section{Acknowledgements}

Waleed Mina, Mena Fouad, Priyanka Trehan, and William M. Briggs.

\section{Ethical approval}

IRB approval was obtained prior to beginning this quality improvement study. No consent was needed for the Hepatitis C antibody testing (this was confirmed by the New York State Department of Health). Patients were not subjected to anything other than routine bloodwork, which they were going to be receiving anyway (this test could be added-on to any gold-top tube lab test in our hospital). All patients were counseled on test results as well. 